\title{
chromoWIZ: a web tool to query and visualize chromosome-anchored genes from cereal and model genomes
}

Thomas Nussbaumer ${ }^{1 \dagger}$, Karl G Kugler $^{1 \dagger}$, Wolfgang Schweiger ${ }^{2}$, Kai C Bader ${ }^{1}$, Heidrun Gundlach', Manuel Spannagl', Naser Poursarebani ${ }^{3}$, Matthias Pfeifer ${ }^{1}$ and Klaus FX Mayer ${ }^{1 *}$

\begin{abstract}
Background: Over the last years reference genome sequences of several economically and scientifically important cereals and model plants became available. Despite the agricultural significance of these crops only a small number of tools exist that allow users to inspect and visualize the genomic position of genes of interest in an interactive manner.

Description: We present chromoWIZ, a web tool that allows visualizing the genomic positions of relevant genes and comparing these data between different plant genomes. Genes can be queried using gene identifiers, functional annotations, or sequence homology in four grass species (Triticum aestivum, Hordeum vulgare, Brachypodium distachyon, Oryza sativa). The distribution of the anchored genes is visualized along the chromosomes by using heat maps. Custom gene expression measurements, differential expression information, and gene-to-group mappings can be uploaded and can be used for further filtering.

Conclusions: This tool is mainly designed for breeders and plant researchers, who are interested in the location and the distribution of candidate genes as well as in the syntenic relationships between different grass species. chromoWIZ is freely available and online accessible at http://mips.helmholtz-muenchen.de/plant/chromoWIZ/index.jsp.
\end{abstract}

Keywords: Cereals, Bread wheat, Barley, Brachypodium, Rice, Comparative genomics

\section{Background}

Since the release of the sequenced genome of Arabidopsis thaliana in 2000 [1], more than 50 plant reference sequences have become available [2]. While the average genome size in Angiosperms is about $6 \mathrm{~Gb}$ [3], sequencing efforts have focused mainly on smaller-sized genomes $(<1 \mathrm{~Gb})$, which serve as models for large and still unsequenced species or on more accessible crop plant genomes such as rice (Oryza sativa). The cereal species of the Pooideae subfamily, including bread wheat (Triticum aestivum), barley (Hordeum vulgare), and rice are among the most important crops and share a high degree of syntenic conservation on a genome-wide level [4,5]. Among the crops, hexaploid bread wheat

\footnotetext{
* Correspondence: k.mayer@helmholtz-muenchen.de

${ }^{\dagger}$ Equal contributors

'Plant Genome and System Biology (PGSB), Helmholtz Center Munich, D-85764 Neuherberg, Germany

Full list of author information is available at the end of the article
}

( $T$. aestivum, $2 \mathrm{n}=6 \mathrm{x}=42$, AABBDD) contains the largest and most complex genome with a size of roughly 17 Gb [6]. Despite its high economic relevance $-20 \%$ of the calories consumed by the world's population derive from bread wheat - its genome has so far not been completely assembled. It has taken several years to provide a reference sequence for even one chromosome (3B, [7]), which by itself exceeds the genome size of rice almost 3 -fold. Recently, shotgun sequencing and flow cytometry provided the basis for a gene annotation of the complete bread wheat genome comprising $\sim 124 \mathrm{k}$ gene models [6]. Furthermore, for selected chromosomes or chromosome arms, a physical map has been established and genetically anchored (e.g. 1A [8,9], 1BS [10], 3B [7,11], $6 \mathrm{~A}$ [12]). For barley an anchored physical map that covers $3.9 \mathrm{~Gb}$ cumulative map length has been released $[13,14]$, including $26 \mathrm{k}$ high-confidence genes and 
comprises shotgun assemblies from three cultivars. Most shotgun contigs have already been anchored by population genetics. This approach, called POPSEQ [15], was also used to improve the anchoring of the physical map [13]. Like bread wheat and barley, Brachypodium (Brachypodium distachyon) also belongs to the Pooideae subfamily within the Poaceae family. It has a relatively small genome $(\sim 300 \mathrm{Mb})$ and has been widely used as a model organism to study the structure and evolution of other grass species [16]. Rice is another important member of the Poaceae family and represents one of the most important staple foods worldwide. To successfully integrate all the different resources, e.g. genetic information and gene expression measurements, for these crop species, heterogeneous datasets need to be combined. Therefore, tools and standards for interlinking anchored datasets are required (reviewed in [17]). One of the approaches for combining heterogeneous datasets is the "GenomeZipper" [4]. It establishes a virtual order of genes in plants without assembled chromosomes by exploiting the highly conserved synteny to smaller, already sequenced genomes. Largesized syntenic regions, together with genetic marker sets enable an anchoring of most genes for larger-sized cereals including e.g. barley [14], rye (Secale cereale) [18] and Aegilops tauschii [19]. Since after the split from their common ancestor, the position of most genes was conserved, this approach provides robust approximations of the gene positions and order [20].

A small number of tools exist that allow users to inspect the genomic position of query genes in target genomes. For barley it is possible to map query sequences by using IPK Viroblast (http://webblast.ipk-gatersleben.de/) or barleymap (http://floresta.eead.csic.es/barleymap/). However, to our knowledge, no web-based tool exists that covers several genomes and allows calculating and visualizing the gene density along the chromosome. This is especially of importance when several dozen genes need to be mapped, e.g. for analyzing a quantitative trait locus (QTL). Transcriptome-oriented studies might reveal a set of gene candidates and the corresponding genomic position supports in removing false-positives gene candidates and defining the genetic or physical location of the QTL. None of the listed tools provide queries based on functional annotation or the integration of expression data. As part of the GenomeZipper, we have previously implemented a module 'chromoWIZ' which was introduced to ease detection of syntenic regions for a yet unassembled genome and several sequenced and assembled genomes including Brachypodium [16], rice [21] and sorghum (Sorghum bicolor) [22]. Here, we describe the web-based version of chromoWIZ along with new features. Originally, chromoWIZ was restricted to local use as part of the GenomeZipper package and allowed a mapping of genes or shotgun contigs of one chromosome or chromosome arm against the reference genomes Brachypodium, rice and sorghum. To find genomic positions for genes of interest, in the latest, web-based version functional annotations and sequence homology can be used to find the corresponding regions within the corresponding genome. For grouped or clustered genes chromoWIZ now visualizes the physical position in a group-wise manner. In its latest version, chromoWIZ integrates the anchoring results of both the International Barley Genome Sequence Consortium (IBSC [14]), and the International Wheat Genome Sequencing Consortium (IWGSC [6]) and allows comparing sequences against the genomes of Brachypodium and rice. This tool is mainly designed for breeders and plant researchers, who are interested in the location and the distribution of candidate genes as well as in the syntenic relationships between different grass species. In order to illustrate the features of chromoWIZ and to explain the basic work-flows, we present different use cases. The application website can be accessed at: http://mips.helmholtz-muenchen. $\mathrm{de} /$ plant/chromoWIZ/index.jsp without any restrictions.

\section{Construction and content}

chromoWIZ runs on a webserver at the PGSB site [23]. The tool's back-end is implemented in the programming language Python. The front-end uses native HTML and Javascript for data visualization and navigation. Mapping information and gene information were collected from the official releases of the Brachypodium, rice, barley and bread wheat genomes [6,14,16,21]. For Brachypodium protein and coding sequences, as well as functional annotation information were collected from the PGSB database [23] using gene models' version 1.2. For barley we integrated the datasets that were provided with the genetically anchored physical map [14], which is hosted at ftp://ftpmips.helmholtz-muenchen.de/plants/ barley/public_data. For bread wheat, gene models from version 2.2 (ftp://ftpmips.helmholtz-muenchen.de/plants/ wheat/IWGSC) were included. The MSU7 annotation has been integrated for rice [21]. More details for the currently used datasets and the corresponding updates are provided on the chromoWIZ web site.

\section{Utility}

\section{Application of chromoWIZ}

chromoWIZ allows visualizing the location of anchored genes along chromosomes on the basis of functional gene annotations, sequence homology or gene lists. So far, the web tool includes the crop species bread wheat ( $T$. aestivum), barley (H. vulgare) and the closely related but much smaller Brachypodium (B. distachyon) and rice (O. sativa) genomes. Anchored genes are clustered 
together along the chromosome in non-overlapping genomic or genetic intervals, referred to as bins. In Brachypodium and rice, every bin represents one megabase $(\mathrm{Mb})$ of non-overlapping chromosomal sequence. For barley $10 \mathrm{Mb}$ and for bread wheat 5 CentiMorgan (cM) intervals are used. Bins are visualized as heat maps to enable an intuitive view along the entire chromosomes. The genomic positions in barley are highlighted relative to the anchored physical BAC contigs which were strung together to form virtual chromosomes. All genes within chromoWIZ are linked to external databases providing additional information on the gene models (e.g. for bread wheat EnsemblePlants http://plants.ensembl. org/Triticum_aestivum/Info/Index). The sequences of tagged genes within a bin can be downloaded in the FASTA format. To obtain the genomic location for genes of interest, referred to as "tagged genes", chromoWIZ provides several search methods (Table 1): By sequence homology a set of query sequences can be mapped against the annotated gene models using nucleotide or protein BLAST searches, requiring a predefined e-value and sequence identity. Alternatively, if known, a list of species-specific gene identifiers can be directly provided instead of sequences. To query families of genes (e.g. genes sharing a specific Gene Ontology (GO) term or PFAM domain $[24,25]$, an annotation-based approach has been included. The distribution of query genes is visualized by heat maps, which depict the relative distribution of the query-matching genes compared to the overall number of genes along the chromosomes. In addition, the overall gene distribution is shown, as the number of anchored genes varies between the different bins. To see whether certain chromosome (-arms) are enriched for tagged genes an enrichment analysis is provided. The significance of over-representation of genes tagged is assessed by a one-sided Fisher's exact test and a Bonferroni adjustment of $\mathrm{P}$ values. Furthermore, labeled groups of genes can be included, e.g. genes being clustered or co-expressed or that were grouped together based on sequence similarity to allow for a group-wise visualization and analysis. The Data Manager is a part of chromoWIZ that enables the upload of various user-specific datasets and performs a validation of input data prior to integration into the chromoWIZ search interface. These data are subsequently only visible for the respective user and available for 24 hours before they are being automatically removed from the servers. Gene expression is an important factor for judging the relevance of candidate genes. In chromoWIZ, by using the Data Manager, users can optionally upload expression values for their genes of interest. Similar to expression data, information about differential expression can be provided. With expression data at hand, functional information can be combined with the genomic positions.

The following use cases illustrate different aspects of chromoWIZ. The first use case describes how candidate genes can be mapped against the reference genome sequences using the gene identifiers, sequence-based searches or functional annotations. The second use case illustrates how a list of genes can be filtered based on their expression or by including information about differential expression. In the third use case we show how chromoWIZ allows highlighting syntenic regions between bread wheat and Brachypodium or barley. In the fourth use case we use published expression data to illustrate how the gene-to-group information can help in refining the genomic position of a resistance QTL. This is granted by transferring data from ancient to recent reference sequences. The fifth use case finally gives an example of how chromoWIZ can be applied for comparative genomic analysis.

Table 1 A variety of search features are provided by chromoWIZ

\begin{tabular}{|c|c|c|}
\hline Search feature & Description & Data needed \\
\hline Sequence similarity & $\begin{array}{l}\text { Genes can be searched using homology either on nucleotide sequence level (BLASTN) or } \\
\text { protein sequence level (BLASTP). }\end{array}$ & - \\
\hline Gene identifier & List of gene identifiers as provided within the genome release. & - \\
\hline $\begin{array}{l}\text { Gene Ontology (GO) } \\
\text { annotation }\end{array}$ & Genes can be searched based on their GO annotation. & - \\
\hline PFAM annotation & Genes can be searched based on their PFAM annotation. & - \\
\hline Expression variation & $\begin{array}{l}\text { Gene expression levels need to vary across conditions in order to filter for interesting genes } \\
\text { as quantified by using the coefficient of variation (sample standard deviation divided by the } \\
\text { sample mean). }\end{array}$ & Expression matrix \\
\hline Presence of expression & The expression has to surpass a custom expression threshold in at least one condition. & Expression matrix \\
\hline Differential expression & Genes have to be in a list of genes being differentially expressed, as provided by the user. & $\begin{array}{l}\text { List of differentially expressed } \\
\text { genes }\end{array}$ \\
\hline Gene clustering & $\begin{array}{l}\text { Genes have to be in a certain group of clustered genes. Clustering information is provided } \\
\text { by the user. }\end{array}$ & Gene to cluster linkage list \\
\hline
\end{tabular}




\section{Use case 1: finding genes using identifiers, sequence similarity or annotations}

One of the very basic functionalities of chromoWIZ is searching and visualizing genes by their identifiers. Given a set of species-specific gene identifiers their genomic position can be highlighted. In case no identifiers are available, an alternative approach is to provide sequence information for the corresponding genes. To illustrate this feature, we use the following example: A list of 19 gene identifiers from Brachypodium, preselected from a particular genomic region, was provided to the search interface (the gene identifiers are given in Additional file 1). chromoWIZ provides two outputs: First a heat map which depicts the number of all anchored genes along the chromosomes per bin (Figure 1A), and secondly, a heat map showing only the anchored genes that meet the query criteria (tagged genes) is shown (Figure 1B). For the given example the corresponding bin (bin9, 9-10 Mb) on chromosome 5 is highlighted. To illustrate the sequencebased search, we first extracted the gene sequences from this bin, by using the FASTA export functionality of chromoWIZ. This set of sequences was then provided to the search interface in order to perform a homology-based search. By only visualizing matches below an e-value of $10 \mathrm{E}-5$, sequence identity of $100 \%$ and by requiring a best bidirectional match (flag 'BBH' has to be set) we again retrieved the bin containing the genes.
Besides the gene identifier and homology-based search, chromoWIZ also offers a search by gene annotation functionalities. A user might be interested in a particular gene family and would like to analyze whether members of that family have increased or decreased copy numbers compared to other genomes. One way to analyze differences in copy numbers is to compare the amount of genes on the basis of protein families (PFAM [25]) or Gene Ontology (GO [24]) terms and chromoWIZ includes annotation information from these sources. In the given example, we aimed at visualizing all genes that are annotated under the Gene Ontology (GO) term GO:0043565 (sequence-specific DNA binding) that e.g. comprises transcription factors. In Brachypodium, we found matches to 349 genes, in bread wheat matches to 421 (732 including genetically unanchored) genes, and in barley we found matches to 225 (340) genes.

Use case 2: filtering for differentially expressed genes and usage of expression constraints

RNA-seq data is commonly used to analyze gene expression on a genome-wide level. It can efficiently be processed by means of analysis pipelines such as Cufflinks [26] or HTSeq [27]. After finding gene candidates based on their expression patterns it is often of interest to explore their respective genomic position. chromoWIZ provides features for combining expression data with

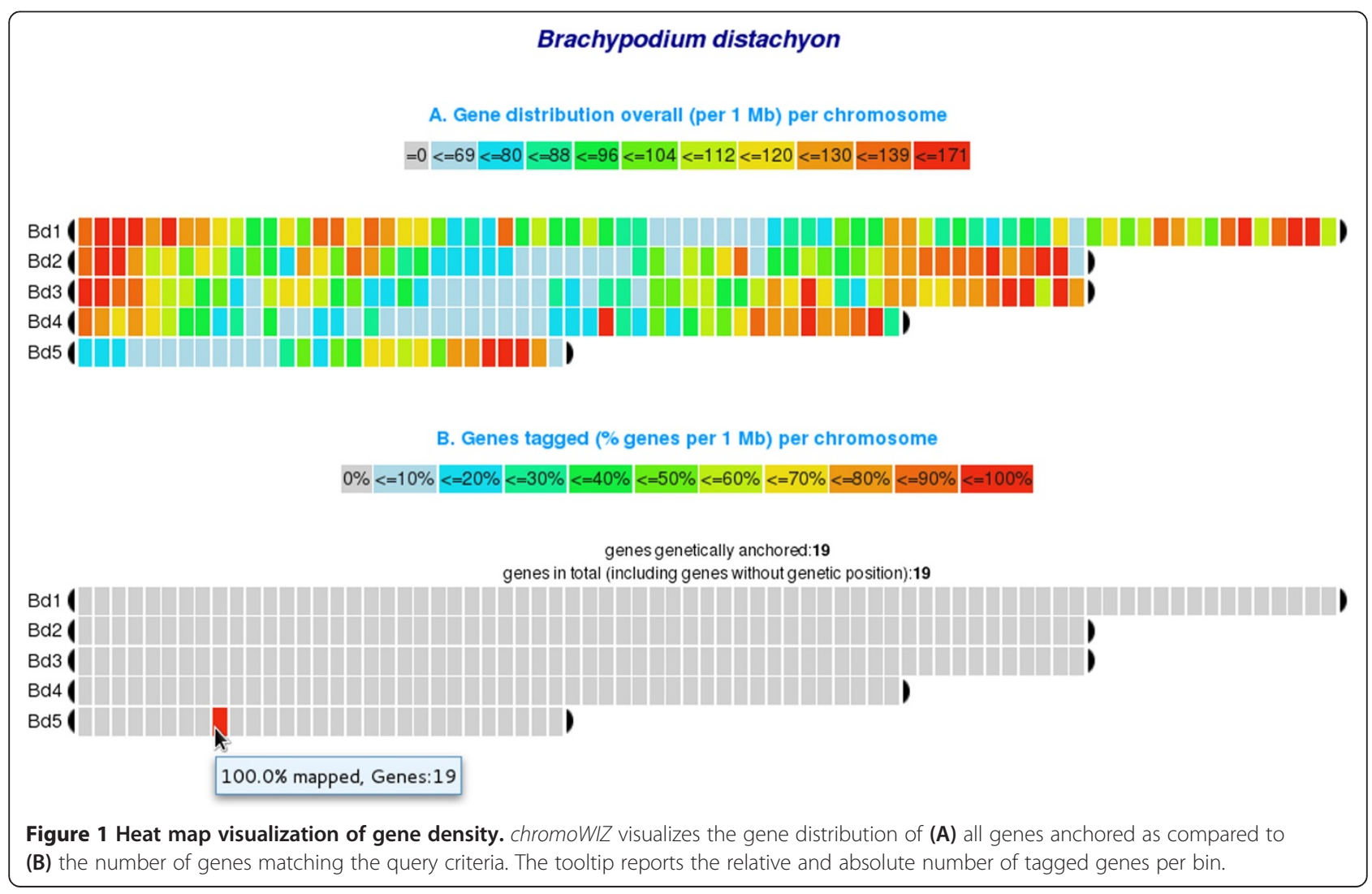


positional information: (i) gene-to-group information can be provided. (ii) lists of differentially expressed genes can be included, and (iii) expression data of all genes can be integrated. Figure 2 shows the Data Manager input and the extended query features on the entry site, which are available once the data sets are included. Gene-to-group information is provided by an input file where the first column contains the gene identifier and the second column defines the group. The differentially expressed genes (DEGs) are provided via an input file that contains the gene identifiers. Also expression information can be provided in a file, where columns represent the conditions of interest. Details about the file formats are given on the chromoWIZ help page. When information about differentially expressed genes is included, the user can specify whether only differentially expressed genes should be queried. If expression information is included, genes can be filtered by two criteria: Either by a 'Minimum expression' criterion, meaning that at least in one condition the expression must exceed a given threshold. Alternatively, to find genes with expression variation across conditions, a user can set a ' $C V$ ' (coefficient of variation, given by dividing the sample standard deviation by the sample mean) filter, to only keep genes with a minimum required $C V$ value.

For illustration we extracted 692 barley transcripts that are differentially expressed between two Tibetan wild barley genotypes in response to low potassium treatment [28]. The transcript sequences as given in Table S3 of [28] (http://www.plosone.org/article/fetchSingleRepresentation.action?uri=info:doi/10.1371/journal. pone.0100567.s009) were mapped against the genetically anchored barley gene models using BLASTN (sequence identity greater than $95 \%$, e-value of $10 \mathrm{E}-10$, $\mathrm{BBH}$ criterion). The 450 matching genes were compiled into a list of differentially expressed genes (Additional file 2) and uploaded by using the Data Manager. When searching barley for anchored differentially expressed genes we obtained 286 hits scattered across the different chromosomes.

\section{Use case 3: pronounced syntenic regions shared in grass species}

chromoWIZ has been repeatedly used to define and refine syntenic regions among related reference genomes $[29,30]$. For illustration, we used gene models of bread wheat chromosome 4A [6] and to initiated a sequence homology search against Brachypodium and barley genes. In total 4,830 genes are annotated on chromosome 4A and the corresponding sequences were extracted and aligned against both genomes using BLASTN (sequence identity of at least $70 \%$ and an e-value of $10 \mathrm{E}-5$, best bidirectional hit). We found matches against chromosomes 1 and 4 in Brachypodium and a rearrangement of an approximately $3 \mathrm{Mb}$ genomic region that was shifted from the short arm of chromosome 1 to the long arm (Figure 3A).

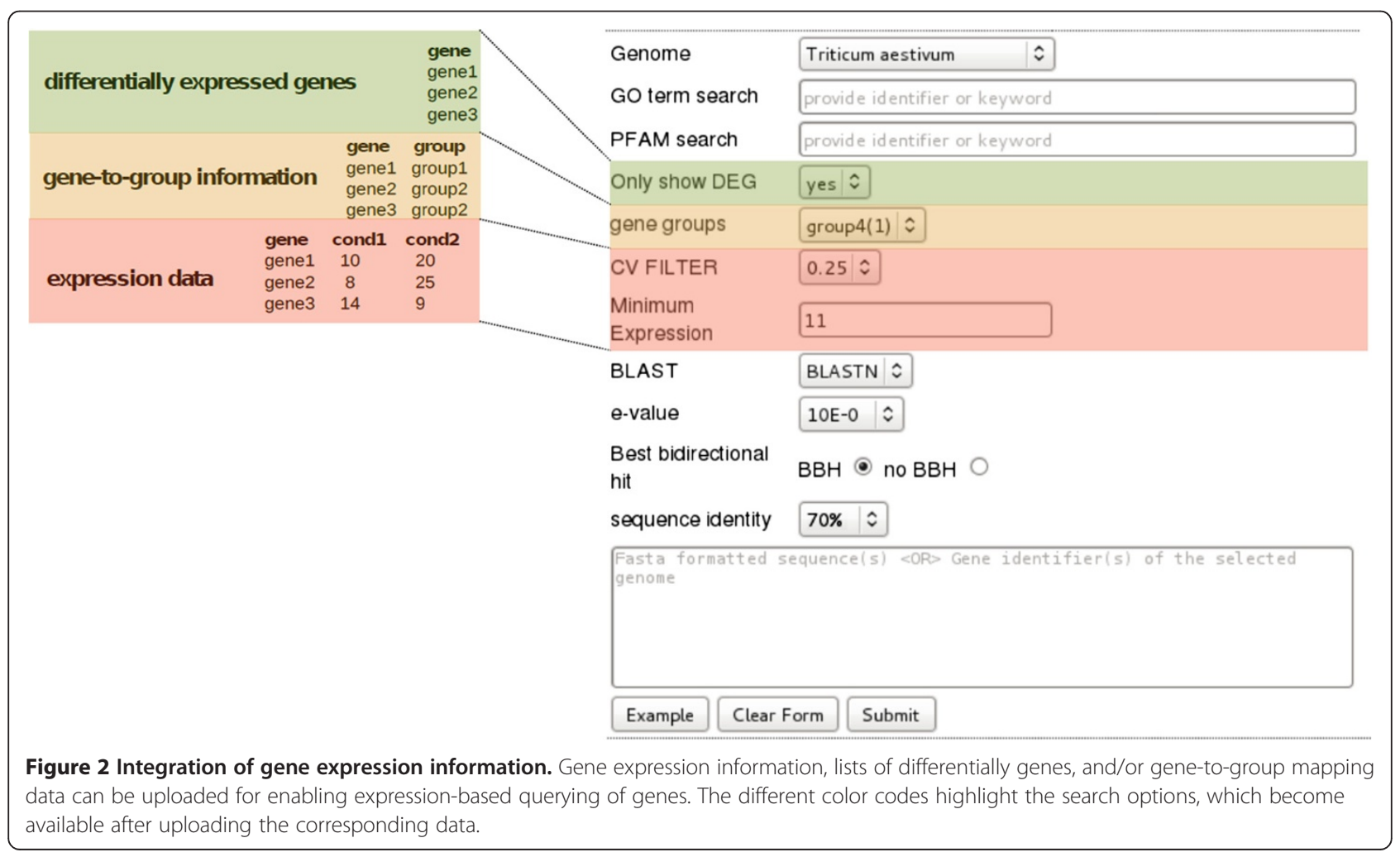




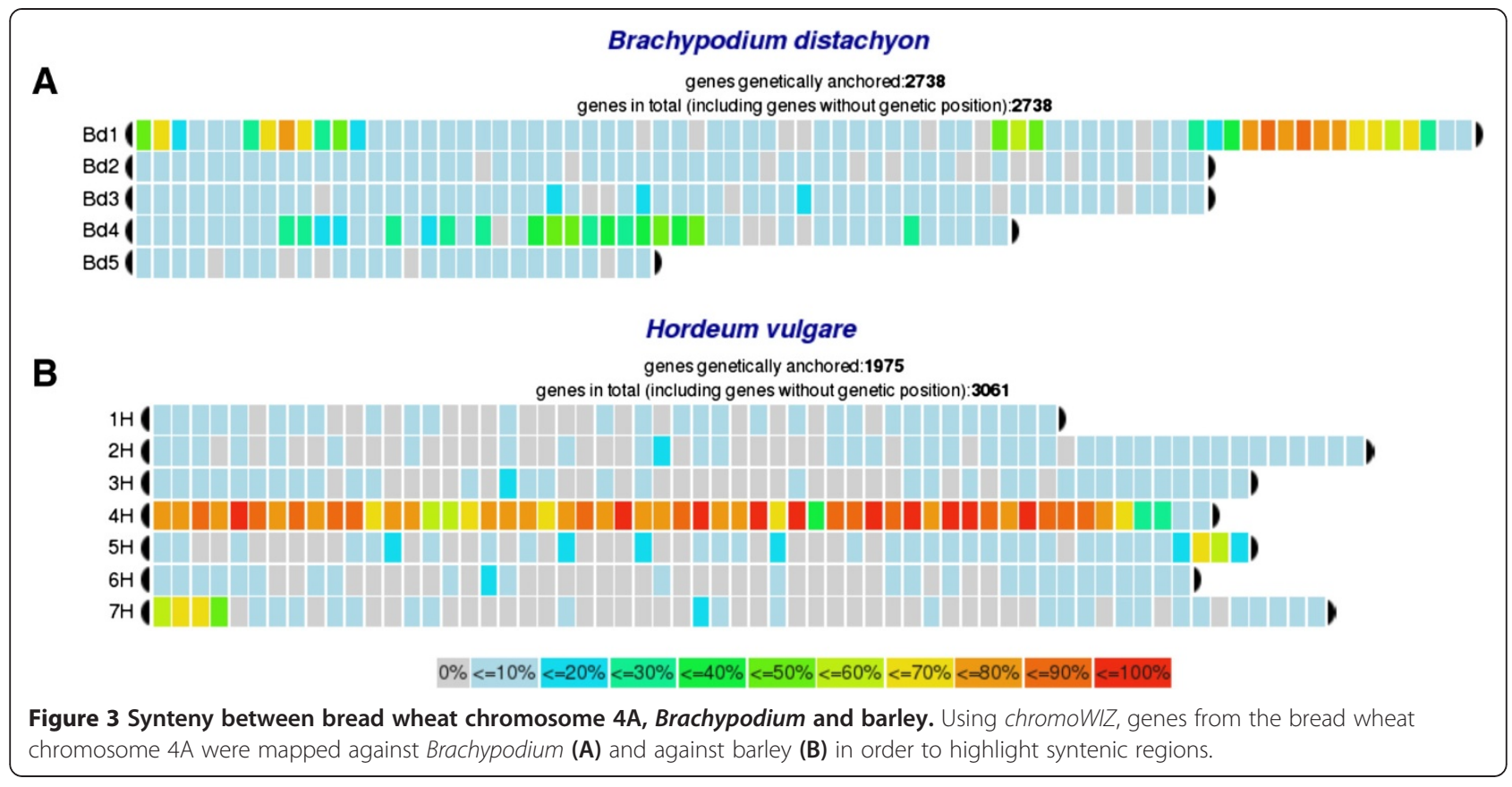

Additionally, in chromosome 4, the centromeric and pericentromeric near regions were tagged. When bread wheat chromosome 4A was compared against barley, besides the largely homeologous chromosome $4 \mathrm{H}$, syntenic regions on chromosome $5 \mathrm{H}$ and chromosome $7 \mathrm{H}$ were found, comprising genomic regions of $40 \mathrm{Mb}$ respectively (Figure 3B). These findings are consistent with the documented chromosome rearrangements of bread wheat chromosome 4A [31].

Use case 4: providing cluster information for tagging genes Clustering genome-wide expression data into meaningful subsets has become a standard procedure in many transcriptome-oriented studies. Several methods enable to perform such a partitioning of data, e.g. by hierarchical clustering, k-means clustering or network-based approaches. chromoWIZ provides support for group-wise analyses as it allows uploading gene-to-group information. The example data for this use case originates from a co-expression network study assessing the effect of fungal pathogens on different bread wheat lines [32]. The five bread wheat lines in this study were characterized by the presence or absence of particular quantitative trait loci (QTL), which confer different resistance levels. This data has been used to infer a co-expression network with the Weighted Correlation Network Analysis approach (WGCNA, [33]). WGCNA can be utilized to find clusters of highly connected genes, so called network modules, based on inferring a correlation-based weighted gene network. After mapping the bread wheat transcriptome data to a 454 sequencing based whole genome assembly [34] and after quantifying the expression using
Cufflinks [26], we observed eight different modules which represented distinct expression patterns containing 3,273 genes in total. One module was of particular interest as the related gene expression depicted a pronounced response to the fungal pathogen. The corresponding nucleotide sequences are given in Additional file 3 . Using chromoWIZ those transcripts were mapped against the bread wheat genome survey sequence [6] by requiring a best bidirectional match and sequence identity of at least 95\%. A significant enrichment for chromosome (-arms) 3B, 5BL, and 7DL was found (Figure 4). This is in support of the experimental setup as one of the major Fusarium head blight resistance QTLs (Fhb1) that segregates between resistant and susceptible lines and is located on the short arm of chromosome 3B [35].

\section{Use case 5: comparative genomics in chromoWIZ for analyzing UDP-gylcosyltransferases}

chromoWIZ can be used to detect homologous genes and their locations in the four cereal and model genomes using the implemented BLAST searches. To illustrate this, we searched for Brachypodium UDP-glycosyltransferases (UGT) homologous genes in rice, barley, and bread wheat. The Brachypodium UGT gene family contains five members of which several encode for the ability to inactivate the mycotoxin deoxynivalenol (Additional file 4) [36]. Deoxynivalenol is a potent inhibitor of protein biosynthesis produced by Fusarium graminearum, which is a pathogen also to wheat and barley [37]. The presence/activity of such UGTs may confirm high resistance. Yet, their identification remains challenging also due to the sheer 


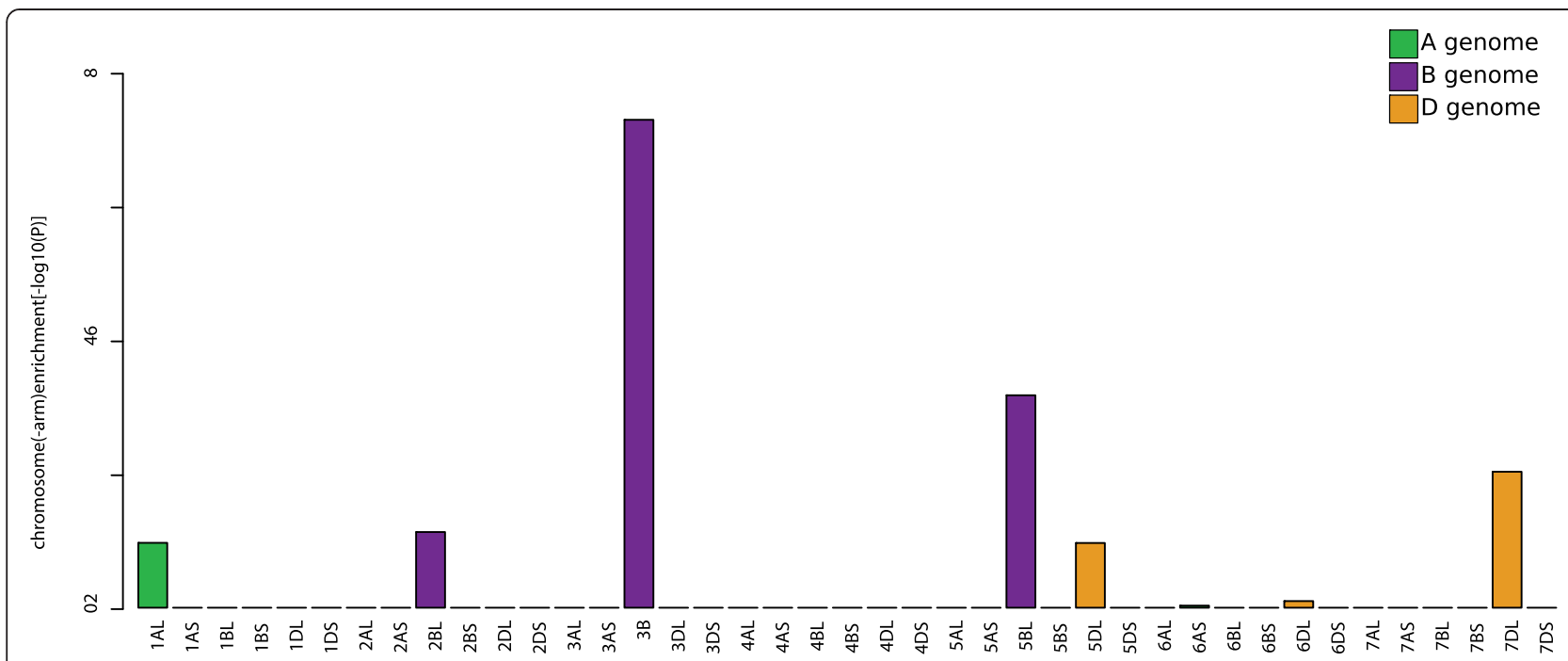

Figure 4 Chromosome (-arm) enrichment of genes responsive to a fungal pathogen. Bread wheat chromosome (-arm) enrichment for genes, which were responsive to Fusarium graminearum. Chromosome (-arms) 3B, 5BL, and 7DL are found to be significantly enriched for these genes.

size of the UGT superfamily, which comprises 178 members in Brachypodium and probably several hundred in bread wheat [36]. chromoWIZ mapped these six genes to the third and fourth bin on chromosome 5 in Brachypodium. In order to find putative orthologous genes, we extracted the sequences and mapped them against rice, bread wheat and barley. In barley, matches were found to the $2 \mathrm{H} \mathrm{(3)} \mathrm{and} 5 \mathrm{H}(1)$ chromosomes using $70 \%$ identity and e-value of $10 \mathrm{E}-5$ as search criterions. In addition a match to a yet genetically unanchored gene was found. In rice, matches on chromosome 4 (8) and chromosome 9 (1) were observed, confirming previous findings [36]. In bread wheat matches to $2 \mathrm{~A}(1), 2 \mathrm{~B}(1), 2 \mathrm{D}(1)$ and $5 \mathrm{~A}(1)$ indicate possible homoeologous gene-clusters on linkage group 2, however most genes (13) did not receive any genetic position yet. No matches were observed for chromosome 3B containing the Fhb1 locus [35], which was previously shown to govern the higher ability to inactivate the toxin [38].

\section{Discussion}

chromoWIZ allows searching for candidate genes and visualizing their density and localizations along chromosomes of selected grass genomes. Genes can be searched by using several options, e.g. by gene identifiers, by functional annotation, by sequence homology search or by gene-to-group mappings. The tool is implemented in a flexible way to ensure that novel genomes or updates of existing genomes can be easily undertaken. Export features are provided and extended functionality is activated if gene expression data or clustering information is provided.
chromoWIZ enables the integration of expression-based information to filter for candidate genes

While there are several tools that provide information, mapping, and visualizations capabilities with respect to syntenic relationships in plant genomes [39,40], there is a lack for tools to query and interactively inspect genetically and physically anchored genes. One of the major advantages of chromoWIZ over other tools such as barleymap (http://floresta.eead.csic.es/barleymap/) or IPK Viroblast (http://webblast.ipk-gatersleben.de/barley/) is that expression data can be included to filter by several criteria and thereby selecting the most relevant genes. In addition, clustering information and gene-to-group mappings such as sets of co-expressed genes, selected gene families and/or differentially expressed genes can be included and independently analyzed. The different datasets can be imported by using the Data Manager as intrinsic part of the chromoWIZ web application. After uploading the data additional filtering and search options appear on the entry page (Table 1 and Figure 2).

\section{chromoWIZ enables transferring previous results to the current reference sequences}

chromoWIZ allows linking gene anchoring information with the annotated gene information and provides access to the gene candidates and their localization as well as to their neighboring genes. With actively ongoing projects and the consequential updates of the reference sequences of bread wheat and barley, data need to be mapped to a common reference sequence to compare previous results against current ones. We demonstrated this approach by using a particular gene co-expression module that comprised the major response of bread wheat genes against a fungal pathogen [32]. As shown in 
use case 4 chromoWIZ allowed transferring previous analysis [32] onto updated resources by mapping from an earlier bread wheat genome draft [34] to more recent chromosome-arm sorted shotgun contigs [6].

\section{chromoWIZ enables to detect larger syntenic blocks within yet unfinished genomes}

For (novel) grass genomes, chromoWIZ can be used to detect and analyze syntenic regions with respect to Brachypodium, rice, barley, and bread wheat. In use case 3, annotated gene models of bread wheat chromosome $4 \mathrm{~A}$ were used to detect syntenic regions in comparison to barley and Brachypodium (Figure 3). This chromosome is of particular interest, because in most cases barley and wheat chromosomes are collinear [4]. For this specific chromosome syntenic regions appeared also on barley chromosomes $5 \mathrm{H}$ and $7 \mathrm{H}$ [31]. Furthermore, when arm sorted chromosome datasets become available for a newly sequenced but not yet assembled genome, chromoWIZ can help to allocate genes to corresponding syntenic regions in barley, rice, bread wheat, and Brachypodium. Thereby, it offers a first glance at the genome structure of these plants, particularly for revealing rearrangements and introgression and to analyze more complex nested syntenic structures.

\section{Conclusions}

chromoWIZ provides a valuable and user-friendly interface to access anchored genes for agriculturally important crops and model genomes. By using the different query options it is possible to flexibly narrow down regions of interest and/or gene candidates. With future updates it is planned to include more species and to extend the range of features prior to allow interactive and integrative searches on evolving large and complex crop plant genomes.

\section{Availability and requirements}

chromoWIZ is freely available without any restrictions at http://mips.helmholtz-muenchen.de/plant/chromoWIZ/ index.jsp.

License: Not required.

Any restrictions to use by non-academics: None.

\section{Availability of supporting data}

The data sets supporting the results of this article are included within the article (and its additional files).

\section{Additional files}

Additional file 1: List of genes for use case 1. List of 19 genes as taken from a particular genomic bin in Brachypodium and used for demonstrating the basic functionality of chromoWIZ.
Additional file 2: Barley genes responsive to low potassium for use case 2. List of barley genes matching transcripts from a study about Tibetan wild barley genotypes under low potassium [28] those were used for integration into the Data Manager.

Additional file 3: List of fungal pathogen-responsive genes for use case 4. List of genes that were clustered together in a Fusarium graminearum responsive network module as reported in [32].

Additional file 4: List of UDP-glycosyltransferases homologs as reported in use case $\mathbf{5}$. Brachypodium genes of the UDP-glycosyltransferases family and their homologous matches to rice, barley, and bread wheat.

\section{Abbreviations}

BBH: Best bidirectional hit; GO: Gene Ontology; IBSC: International Barley Genome Sequencing Consortium; IWGSC: International Wheat Genome Sequencing Consortium; POPSEQ: Anchoring and ordering NGS contig assemblies by population sequencing; QTL: Quantitative trait loci; UGT: UDP-dependent glycosyltransferases; WGCNA: Weighted Correlation Network Analysis.

\section{Competing interests}

The authors declare that they have no competing interests.

\section{Authors' contributions}

$H G, T N$, and KFXM initiated the first version of the software. TN, KGK, and KCB implemented the software. KGK, TN, WS, NP, MS, MP, and KFXM drafted and designed the use cases. TN, KGK, WS, and KFXM drafted and wrote the manuscript. All authors approved the final version of the manuscript.

\section{Acknowledgements}

We gratefully acknowledge the Funding by the Deutsche

Forschungsgemeinschaft (DFG) SFB 924 to KFXM and by the Austrian Science Fund (FWF) special research project F37 (F3705, F3711).

\section{Author details}

1Plant Genome and System Biology (PGSB), Helmholtz Center Munich, D-85764 Neuherberg, Germany. ${ }^{2}$ Institute for Biotechnology in Plant Production, IFA-Tulln, University of Natural Resources and Life Sciences, A-3430 Tulln, Austria. '2 Leibniz Institute of Plant Genetics and Crop Plant Research (IPK), OT Gatersleben, Corrensstraße 3, D-06466 Stadt Seeland, Germany.

Received: 1 September 2014 Accepted: 24 November 2014

Published online: 10 December 2014

\section{References}

1. Arabidopsis Genome Initiative: Analysis of the genome sequence of the flowering plant Arabidopsis thaliana. Nature 2000, 408(6814):796-815.

2. Michael TP, Jackson S: The first 50 plant genomes. Plant Genome 2013, 6(2). https://www.crops.org/publications/tpg/articles/6/2/ plantgenome2013.03.0001in.

3. Morrell PL, Buckler ES, Ross-Ibarra J: Crop genomics: advances and applications. Nat Rev Genet 2011, 13(2):85-96.

4. Mayer KF, Martis M, Hedley PE, Simkova H, Liu H, Morris JA, Steuernagel B, Taudien S, Roessner S, Gundlach H, Kubalakova M, Suchankova P, Murat F, Felder M, Nussbaumer T, Graner A, Salse J, Endo T, Sakai H, Tanaka T, Itoh T, Sato K, Platzer M, Matsumoto T, Scholz U, Dolezel J, Waugh R, Stein N: Unlocking the barley genome by chromosomal and comparative genomics. Plant Cell 2011, 23(4):1249-1263.

5. Bolot S, Abrouk M, Masood-Quraishi U, Stein N, Messing J, Feuillet C, Salse J: The 'inner circle' of the cereal genomes. Curr Opin Plant Biol 2009, 12(2):119-125.

6. International Wheat Genome Sequencing Consortium: A chromosome-based draft sequence of the hexaploid bread wheat (Triticum aestivum) genome. Science 2014, 345(6194):1251788.

7. Choulet F, Alberti A, Theil S, Glover N, Barbe V, Daron J, Pingault L, Sourdille P, Couloux A, Paux E, Leroy P, Mangenot S, Guilhot N, Le Gouis J, Balfourier F, Alaux M, Jamilloux V, Poulain J, Durand C, Bellec A, Gaspin C, Safar J, Dolezel J, Rogers J, Vandepoele K, Aury JM, Mayer K, Berges H, Quesneville $H$, Wincker $P$, et al: Structural and functional partitioning of bread wheat chromosome 3B. Science 2014, 345(6194):1249721. 
8. Breen J, Wicker T, Shatalina M, Frenkel Z, Bertin I, Philippe R, Spielmeyer W, Simkova H, Safar J, Cattonaro F, Scalabrin S, Magni F, Vautrin S, Berges H, International Wheat Genome Sequencing C, Paux E, Fahima T, Dolezel J, Korol A, Feuillet C, Keller B: A physical map of the short arm of wheat chromosome 1A. PLoS One 2013, 8(11):e80272

9. Lucas SJ, Akpinar BA, Kantar M, Weinstein Z, Aydinoglu F, Safar J, Simkova H, Frenkel Z, Korol A, Magni F, Cattonaro F, Vautrin S, Bellec A, Berges H, Dolezel J, Budak H: Physical mapping integrated with syntenic analysis to characterize the gene space of the long arm of wheat chromosome $1 \mathrm{~A}$. PLoS One 2013, 8(4):e59542.

10. Raats D, Frenkel Z, Krugman T, Dodek I, Sela H, Simkova H, Magni F, Cattonaro F, Vautrin S, Berges H, Wicker T, Keller B, Leroy P, Philippe R, Paux E, Dolezel J, Feuillet C, Korol A, Fahima T: The physical map of wheat chromosome $1 \mathrm{BS}$ provides insights into its gene space organization and evolution. Genome Biol 2013, 14(12):R138.

11. Paux E, Sourdille P, Salse J, Saintenac C, Choulet F, Leroy P, Korol A, Michalak M, Kianian S, Spielmeyer W, Lagudah E, Somers D, Kilian A, Alaux M, Vautrin S, Berges H, Eversole K, Appels R, Safar J, Simkova H, Dolezel J, Bernard M, Feuillet C: A physical map of the 1-gigabase bread wheat chromosome 3B. Science 2008, 322(5898):101-104

12. Poursarebani N, Nussbaumer T, Simkova H, Safar J, Witsenboer $H$, van Oeveren J, Dolezel J, Mayer KF, Stein N, Schnurbusch T: Whole-genome profiling and shotgun sequencing delivers an anchored, gene-decorated, physical map assembly of bread wheat chromosome 6A. Plant Journal 2014, 79(2):334-347

13. Ariyadasa $R$, Mascher $M$, Nussbaumer $T$, Schulte $D$, Frenkel $Z$, Poursarebani N, Zhou R, Steuernagel B, Gundlach H, Taudien S, Felder M, Platzer M, Himmelbach A, Schmutzer T, Hedley PE, Muehlbauer GJ, Scholz U, Korol A, Mayer KF, Waugh R, Langridge P, Graner A, Stein N: A sequence-ready physical map of barley anchored genetically by two million single-nucleotide polymorphisms. Plant Physiol 2014, 164(1):412-423.

14. International Barley Genome Sequencing Consortium, Mayer KF, Waugh R, Brown JW, Schulman A, Langridge P, Platzer M, Fincher GB, Muehlbauer GJ, Sato K, Close TJ, Wise RP, Stein N: A physical, genetic and functional sequence assembly of the barley genome. Nature 2012, 491(7426):711-716.

15. Mascher M, Muehlbauer GJ, Rokhsar DS, Chapman J, Schmutz J, Barry K, Munoz-Amatriain M, Close TJ, Wise RP, Schulman AH, Himmelbach A, Mayer KF, Scholz U, Poland JA, Stein N, Waugh R: Anchoring and ordering NGS contig assemblies by population sequencing (POPSEQ). Plant J 2013 76(4):718-727

16. International Brachypodium Initiative: Genome sequencing and analysis of the model grass Brachypodium distachyon. Nature 2010, 463(7282):763-768

17. Spannagl M, Martis MM, Pfeifer M, Nussbaumer T, Mayer K: Analysing complex Triticeae genomes—concepts and strategies. Plant Methods 2013, 9(1):35

18. Martis MM, Zhou R, Haseneyer G, Schmutzer T, Vrana J, Kubalakova M, Konig S, Kugler KG, Scholz U, Hackauf B, Korzun V, Schon CC, Dolezel J, Bauer E, Mayer KF, Stein N: Reticulate evolution of the rye genome. Plant Cell 2013, 25(10):3685-3698.

19. Jia J, Zhao S, Kong X, Li Y, Zhao G, He W, Appels R, Pfeifer M, Tao Y, Zhang X, Jing R, Zhang C, Ma Y, Gao L, Gao C, Spannagl M, Mayer KF, Li D, Pan S, Zheng F, Hu Q, Xia X, Li J, Liang Q, Chen J, Wicker T, Gou C, Kuang H, He G, Luo $Y$, et al: Aegilops tauschii draft genome sequence reveals a gene repertoire for wheat adaptation. Nature 2013, 496(7443):91-95.

20. Poursarebani N, Ariyadasa R, Zhou R, Schulte D, Steuernagel B, Martis MM Graner A, Schweizer P, Scholz U, Mayer K, Stein N: Conserved synteny-based anchoring of the barley genome physical map. Funct Integr Genomics 2013, 13(3):339-350

21. International Rice Genome Sequencing Project: The map-based sequence of the rice genome. Nature 2005, 436(7052):793-800

22. Paterson AH, Bowers JE, Bruggmann R, Dubchak I, Grimwood J, Gundlach H, Haberer G, Hellsten U, Mitros T, Poliakov A, Schmutz J, Spannagl M, Tang H, Wang X, Wicker T, Bharti AK, Chapman J, Feltus FA, Gowik U, Grigoriev IV, Lyons E, Maher CA, Martis M, Narechania A, Otillar RP, Penning BW, Salamov AA, Wang Y, Zhang L, Carpita NC, et al: The Sorghum bicolor genome and the diversification of grasses. Nature 2009, 457(7229):551-556.

23. Nussbaumer T, Martis MM, Roessner SK, Pfeifer M, Bader KC, Sharma S, Gundlach H, Spannagl M: MIPS PlantsDB: a database framework for comparative plant genome research. Nucleic Acids Res 2013, 41(Database issue):D1144-D1151.

24. Ashburner M, Ball CA, Blake JA, Botstein D, Butler H, Cherry JM, Davis AP, Dolinski K, Dwight SS, Eppig JT, Harris MA, Hill DP, Issel-Tarver L, Kasarskis A
Lewis S, Matese JC, Richardson JE, Ringwald M, Rubin GM, Sherlock G: Gene ontology: tool for the unification of biology. Gene Ontol Consortium Nat Genet 2000, 25(1):25-29.

25. Finn RD, Bateman A, Clements J, Coggill P, Eberhardt RY, Eddy SR, Heger A, Hetherington K, Holm L, Mistry J, Sonnhammer EL, Tate J, Punta M: Pfam: the protein families database. Nucleic Acids Res 2014, 42(Database issue):D222-D230.

26. Trapnell C, Roberts A, Goff L, Pertea G, Kim D, Kelley DR, Pimentel H, Salzberg SL, Rinn JL, Pachter L: Differential gene and transcript expression analysis of RNA-seq experiments with TopHat and Cufflinks. Nat Protoc 2012, 7(3):562-578.

27. Anders S, Pyl PT, Huber W: HTSeq-a Python framework to work with highthroughput sequencing data. Bioinformatics 2014. http://www.biorxiv.org/ content/biorxiv/early/2014/02/20/002824.full.pdf.

28. Zeng J, He X, Wu D, Zhu B, Cai S, Nadira UA, Jabeen Z, Zhang G: Comparative transcriptome profiling of two Tibetan wild barley genotypes in responses to low potassium. PLoS One 2014, 9(6):e100567.

29. Kopecky D, Martis M, Cihalikova J, Hribova E, Vrana J, Bartos J, Kopecka J, Cattonaro F, Stoces S, Novak P, Neumann P, Macas J, Simkova H, Studer B, Asp T, Baird JH, Navratil P, Karafiatova M, Kubalakova M, Safar J, Mayer K, Dolezel J: Flow sorting and sequencing meadow fescue chromosome 4F. Plant Physiol 2013, 163(3):1323-1337.

30. Pfeifer M, Martis M, Asp T, Mayer KF, Lubberstedt T, Byrne S, Frei U, Studer B: The perennial ryegrass GenomeZipper: targeted use of genome resources for comparative grass genomics. Plant Physio/ 2013, 161(2):571-582

31. Hernandez P, Martis M, Dorado G, Pfeifer M, Galvez S, Schaaf S, Jouve N, Simkova H, Valarik M, Dolezel J, Mayer KF: Next-generation sequencing and syntenic integration of flow-sorted arms of wheat chromosome $4 \mathrm{~A}$ exposes the chromosome structure and gene content. Plant J 2012, 69(3):377-386

32. Kugler KG, Siegwart G, Nussbaumer T, Ametz C, Spannagl M, Steiner B, Lemmens M, Mayer KF, Buerstmayr H, Schweiger W: Quantitative trait loci-dependent analysis of a gene co-expression network associated with Fusarium head blight resistance in bread wheat (Triticum aestivum L.). BMC Genomics 2013, 14:728.

33. Langfelder $P$, Horvath S: WGCNA: an R package for weighted correlation network analysis. BMC Bioinformatics 2008, 9:559.

34. Brenchley R, Spannagl M, Pfeifer M, Barker GL, D'Amore R, Allen AM, McKenzie N, Kramer M, Kerhornou A, Bolser D, Kay S, Waite D, Trick M, Bancroft I, Gu Y, Huo N, Luo MC, Sehgal S, Gill B, Kianian S, Anderson O, Kersey P, Dvorak J, McCombie WR, Hall A, Mayer KF, Edwards KJ, Bevan MW, Hall N: Analysis of the bread wheat genome using whole-genome shotgun sequencing. Nature 2012, 491(7426):705-710.

35. Cuthbert PA, Somers DJ, Thomas J, Cloutier S, Brule-Babel A: Fine mapping Fhb1, a major gene controlling fusarium head blight resistance in bread wheat (Triticum aestivum L.). TAG Theor App/ Genet 2006, 112(8):1465-1472.

36. Schweiger W, Pasquet JC, Nussbaumer T, Paris MP, Wiesenberger G, Macadre C, Ametz C, Berthiller F, Lemmens M, Saindrenan P, Mewes HW Mayer KF, Dufresne M, Adam G: Functional characterization of two clusters of Brachypodium distachyon UDP-glycosyltransferases encoding putative deoxynivalenol detoxification genes. Mol Plant Microbe Interact 2013, 26(7):781-792

37. Desjardins AE: Fusarium Mycotoxins: Chemistry, Genetics and Biology. APS Press; 2006.

38. Lemmens M, Scholz U, Berthiller F, Dall'Asta C, Koutnik A, Schuhmacher R, Adam G, Buerstmayr H, Mesterhazy A, Krska R, Ruckenbauer P: The ability to detoxify the mycotoxin deoxynivalenol colocalizes with a major quantitative trait locus for Fusarium head blight resistance in wheat. Mol Plant Microbe Interact 2005, 18(12):1318-1324.

39. Revanna KV, Munro D, Gao A, Chiu CC, Pathak A, Dong Q: A web-based multi-genome synteny viewer for customized data. BMC Bioinformatic 2012, 13:190.

40. Soderlund C, Bomhoff M, Nelson WM: SyMAP v3.4: a turnkey synteny system with application to plant genomes. Nucleic Acids Res 2011, 39(10):e68.

\section{doi:10.1186/s12870-014-0348-6}

Cite this article as: Nussbaumer et al:: chromoWIZ: a web tool to query and visualize chromosome-anchored genes from cereal and model genomes. BMC Plant Biology 2014 14:348. 